\title{
Subtle visuomotor difficulties in preclinical Alzheimer's disease
}

\author{
Maria A. Mollica', Jordi Navarra ${ }^{2}$, Irune Fernández-Prieto ${ }^{2,3,4}$, \\ Jaume Olives', Adrià Tort', Natalia Valech', Nina Coll-Padrós', \\ José L. Molinuevo ${ }^{1,5}$ and Lorena Rami ${ }^{1,5 *}$ \\ 'Alzheimer's Disease and Other Cognitive Disorders Unit, Neurology Service, \\ Hospital Clínic, Barcelona, Spain \\ ${ }^{2}$ Experimental Psychology and Brain Disorders Laboratory, Sant Joan de Deu \\ Healthcare Park, Sant Joan de Deu Hospital, Barcelona, Spain \\ ${ }^{3}$ Biomedical Network Research Centre on Mental Health (CIBERSAM), Madrid, Spain \\ ${ }^{4}$ Institute for Brain, Cognition and Behaviour (IR3C), Department of Basic Psychology, \\ Faculty of Psychology, University of Barcelona, Barcelona, Spain \\ ${ }^{5}$ August Pi i Sunyer Biomedical Research Institute (IDIBAPS), Barcelona, Spain
}

Background. Individuals with preclinical Alzheimer's disease (Pre-AD) present nonimpaired cognition, as measured by standard neuropsychological tests. However, detecting subtle difficulties in cognitive functions may be necessary for an early diagnosis and intervention.

Objectives. A new computer-based visuomotor coordination task (VMC) was developed to investigate the possible presence of early visuomotor difficulties in Pre-AD individuals. Associations between VMC task performance and $A D$ biomarkers were studied. The influence of ApoE status on participants' performance was addressed, as well as the relationship between performance and subjective cognitive decline (SCD).

Methods. Sixty-six cognitively normal $(C N)$ elders (I9 Pre-AD and 47 control participants [CTR]) and I5 patients with AD performed the VMC task, which consisted in executing visually guided goal-directed movements that required the coordination of the visual and motor systems. All participants underwent $A p o E$ analysis and lumbar puncture. $\mathrm{CN}$ participants also completed an extensive standard neuropsychological battery.

Results. Despite presenting normal cognition in standard tests, Pre-AD participants exhibited higher response times (RTs) to complete the VMC task than CTR $(p<.01)$. Besides, patients with AD showed higher RTs than CTR $(p<.001)$ and Pre-AD $(p<.05)$, and more errors than CTR $(p<.005)$. RTs in ApoE4 carriers were higher than that observed in ApoE4 noncarriers $(p<.01)$. In CN individuals, RTs were related to amyloid $\beta$-protein 42 (AB42) biomarker $(p<.0 \mathrm{I})$ and informant-rated SCD $(p<.0 \mathrm{I})$.

Conclusions. The VMC task is able to discriminate Pre-AD from CTR individuals. Moreover, VMC results are associated with $A B 42$ levels in $C N$ individuals, suggesting that visuomotor dysfunction may be a sensitive marker of Pre-AD.

*Correspondence should be addressed to Lorena Rami, Alzheimer's Disease and Other Cognitive Disorders Unit, Neurology Service, Hospital Clínic Villarroel I 70, Barcelona 08036, Spain (email: Irami@clinic.ub.es).

The first two authors contributed equally to the present study. 
An early identification of individuals at risk for Alzheimer's disease (AD) is crucial for developing strategies for an adequate therapeutic prevention. According to recent recommendations (Sperling et al., 2011), the preclinical stages of AD (Pre-AD) can be identified in cognitively normal $(\mathrm{CN})$ individuals when the underlying AD pathophysiological processes are observed in the absence of apparent clinical symptoms. The biomarker model by Jack et al. (2010) postulates that biomarkers related to brain amyloidosis, such as the reduction of amyloid $\beta$-protein 42 (AB42) levels in cerebrospinal fluid (CSF) as well as increased amyloid tracer retention on positron emission tomography, could be the first pathophysiological anomalies to appear in Pre-AD individuals, long before the appearance of neuronal injury. The preclinical phase of the disease lasts for years and usually remains undetected prior to significant cognitive impairment (Jack et al., 2012).

By definition, Pre-AD individuals present normal cognitive functions according to existing standard neuropsychological instruments until they meet criteria for mild cognitive impairment (MCI). However, new computerized tests may provide more sensitive measures than traditional tests, designed for patients who often show pronounced cognitive impairment (Rentz et al., 2013). Recently-developed experimental memory tasks, for example, have shown a higher sensitivity than standard neuropsychological tests in identifying cognitive changes in $\mathrm{CN}$ individuals at risk of developing $\mathrm{AD}$ (Parra et al., 2011; Rentz et al., 2011). Thus, detecting any subtle cognitive difficulty using new computerized measures could be crucial in the near future to detect candidates for treatment during the preclinical stages of $\mathrm{AD}$.

$\mathrm{AD}$ is typically associated with hippocampal damage (La Joie et al., 2012). However, functional anomalies have been reported in the posterior parietal association areas at very early stages of the disease, including the Pre-AD phase (Hedden et al., 2009; Mandal, Joshi, \& Saharan, 2012; Rami et al., 2012). The posterior parietal cortex is known to play an important role in transforming visuospatial information into goal-directed actions, and its connection with the frontal lobe underlies many visuomotor functions (Corbetta \& Shulman, 2002; Culham \& Valyear, 2006). While the involvement of these brain networks in $\mathrm{AD}$ is well documented, the possible visuomotor dysfunction caused by this functional anomaly has not yet been investigated.

Performance on classical neuropsychological tests assessing visuomotor components was found to be predictive of AD in a few recent studies (Donohue et al., 2014; Ewers et al., 2014; Snitz et al., 2013). On the other hand, cross-sectional studies failed at finding any significant relationship between these classical measures and $\mathrm{AD}$ biomarkers in $\mathrm{CN}$ individuals (Hedden, Oh, Younger, \& Patel, 2013). Considering these evidences, we believe that neuropsychological instruments for assessing visuomotor components may not be useful to capture possible subtle alterations in these functions in Pre-AD. Consequently, it is crucial to develop new computer-based measures sensitive enough to reveal fine-grained differences, for example in visuomotor performance, between Pre-AD individuals and cognitively normal elders with a normal $\mathrm{AD}$ biomarker profile.

Previous studies suggest that visuomotor dysfunction may be one of the first clinical symptoms to appear in patients with AD (Tippett, Sergio, \& Black, 2012; Verheij et al., 2012). Along the same lines, recent work has identified visuomotor impairment in highrisk $\mathrm{AD}$ individuals (classified on the basis of their $\mathrm{AD}$ family history or diagnosis of $\mathrm{MCI}$ ), suggesting that it could be a potential marker of AD (Hawkins \& Sergio, 2014). However, the previously identified AD biomarkers, such as amyloid and neuronal injury, were not considered in this previous study. Thus, it is critical to analyse the possible relationship 
between these specific $\mathrm{AD}$ biomarkers and subtle visuomotor difficulties (as measured in computer-based tasks).

In addition to the biological markers, another important factor that may influence cognition at early stages of $\mathrm{AD}$ is the apolipoprotein $\mathrm{E}$ (ApoE) genotype. Forty to 65\% of patients with $\mathrm{AD}$ are ApoE4 carriers, thus making it the major genetic risk factor for sporadic late-onset $\mathrm{AD}$. This has been confirmed in a recent genome-wide association study (GWAS; Lambert et al., 2013). The possible influence of ApoE4 in the early cognitive changes associated with $\mathrm{AD}$ is a relevant issue (Mormino et al., 2014) and trials with asymptomatic ApoE4 carriers, and, in particular, homozygous ones, are under development.

Furthermore, the report of subjective cognitive decline (SCD), understood as the perceived experience of cognitive deterioration, has increasingly been considered as a potential predictor of AD (Jessen et al., 2014). Previous studies have argued that SCD reports are associated with poorer objective cognitive performance and with $\mathrm{AD}$ biomarkers in a CN population (Amariglio et al., 2012; Perrotin, Mormino, Madison, Hayenga, \& Jagust, 2012). Thus, it may also be interesting to analyse how SCD is related to actual performance on new computer-based cognitive tasks.

The aim of the present work was to investigate the possible presence of early visuomotor difficulties in Pre-AD individuals (as compared with a group of cognitively normal elders with normal AD biomarkers profile). For this purpose, a new computerbased visuomotor coordination task (VMC) was developed. Possible associations between VMC task performance and AD biomarkers were studied. The influence of ApoE status on participants' performance in this task was addressed, as well as the relationship between VMC task performance and SCD reports.

\section{Materials and methods}

\section{Participants}

A total of 81 participants were recruited from a memory clinic. The study was approved by the hospital ethics committee, and all participants gave written informed consent prior to enrolment. Sixty-six participants with normal cognition (their scores falling within $1.5 S D$ from normative means in an extensive neuropsychological battery; see below) were recruited for this study. These cognitively normal $(\mathrm{CN})$ participants were further divided into two groups: A control group (CTR) of 47 participants with a normal CSF profile (AB42 $>550 \mathrm{pg} / \mathrm{ml}$, total tau [tau] $<450 \mathrm{pg} / \mathrm{ml}$ and tau phosphorylated [p-tau] $<75 \mathrm{pg} /$ $\mathrm{ml}$ levels); a group of 19 preclinical participants (Pre-AD group) with low levels of CSF AB42 $(<550 \mathrm{pg} / \mathrm{ml})$. Four of these 19 Pre-AD individuals (21\%) also presented abnormal levels of tau and/or p-tau. In addition, 15 patients were included in a third group of patients with $\mathrm{AD}$. This group included participants that met NIA-AA criteria (Jack et al., 2012) for $\mathrm{AD}$ as they had abnormal amyloid and injury markers. The presence of a major psychiatric or neurological diagnosis and/or any serious or unstable medical condition was considered as exclusion criteria.

\section{Visuomotor coordination task}

The VMC task was divided into four blocks containing 20 trials each. Participants were instructed to use their dominant hand on the first two blocks and their nondominant hand in the last two. All trials started with the participants' right/left index finger resting on a 
small platform located in front of the response keyboard. A fixation point was presented at the beginning of each trial on the centre of screen for $1000 \mathrm{~ms}$. Subsequently, the target represented by a green square appeared on the computer screen, randomly in one of 5 different positions (Figure 1) for $200 \mathrm{~ms}$. Participants were instructed to press one of the five green keys available on the keyboard according to the on-screen target position with their index finger as fast and accurately as possible and to return to the original resting position. The position of these five keys mimicked, in a modified computer keyboard, the location of the five squares indicating the target position on the computer screen. The instructions were both given by the experimenter and displayed on the monitor.

The visual 'go' (and target position) signal consisted of one green square $(120 \times 120$ pixels) appearing on a black background on a 19-inch computer screen (HP Compaq LA 1956X Monitor, $75 \mathrm{~Hz}$ ). The response buttons were made of hard foam and were also green on a black background. The 'starting/resting-point' rectangle (also made of foam) was attached to the response keyboard (on its lower central part; Figure 1). DMDX presentation software (Forster \& Forster, 2003), running on an Intel Core computer, was used to present the stimuli and record the participants' RTs (the response time's mean that the subject spends in responding to each stimulus). The RTs' means were calculated considering correct responses exclusively. The experimental sessions were conducted in a quiet room at the memory clinic, where participants sat down in front of the monitor at a distance of $60 \mathrm{~cm}$ approximately.

\section{Cognitive, psychological, and SCD assessment}

All CN participants underwent a complete 2-hr neuropsychological battery, performed by two trained neuropsychologists. Vocabulary from Wechsler Adult Intelligence Scales III (WAIS-III; Wechsler, 1997) was used as a measure of verbal intelligence. Episodic verbal memory was assessed by the Free and Cued Selective Reminding Test (FCSRT; Buschke, 1984). Semantic memory was assessed by a semantic verbal fluency

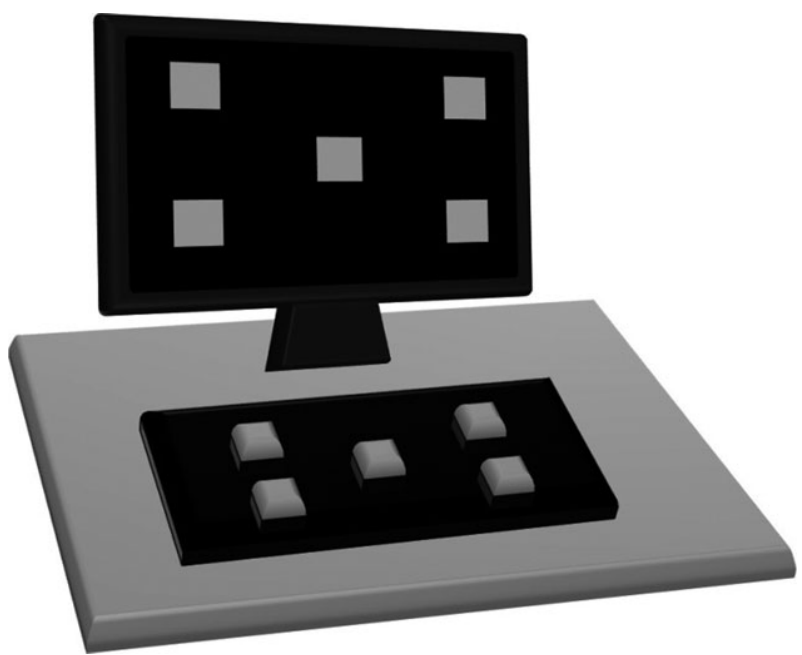

Figure I. Schematic representation of the VMC task setting. The participants were instructed to press one of five different buttons, as previously indicated by a visual 'go' signal (that could appear in different positions) as fast and accurately as possible. 
test (animals), and the Boston Naming Test (BNT; Kaplan, Goodglass, \& Weintraub, 1983) was used to assess language (confrontation naming). Visual perception was assessed by means of the number location and the object decision of the Visual Object and Space Perception (VOSP; Warrington \& James, 1986) battery, and by the block design test of WAIS-III. Visuomotor and executive functions were assessed by Digit Symbol Coding of WAIS-III, by Symbol Digit Modalities Test (SDMT; Smith, 1982), and by the Trail Making Test (TMT), parts A and B (Reitan \& Wolfson, 1985). Inhibition of automatic response was assessed by the interference subtest of the Stroop colour-word (Golden, 1978), and finally, short-term memory was assessed by the forward and backward Digit Span from the WAIS-III. Patients with AD performed a subset of the neuropsychological battery including FCSRT, semantic fluency, number location (VOSP), TMT-A, and Digit Span. For all neuropsychological tests, normalized scalar scores for Spanish population were used (Peña-Casanova et al., 1997).

The Hospital Anxiety and Depression Scale (HADS; Zigmond \& Snaith, 1983) was used to determine the levels of anxiety and depression. The HADS is a fourteen item scale. Seven of the items relate to anxiety and seven relate to depression.

The Subjective Cognitive Decline Questionnaire (SCD-Q; Rami et al., 2014) was also administrated in a subset of the sample (26 CTR, 15 Pre-AD and $11 \mathrm{AD}$ participants) for the assessment of SCD. SCD-Q contains two parts: Part I (SCD-Q MyCog) is completed by the subjects themselves and indicates their self-rated cognitive decline. Part II (SCD-Q TheirCog) is completed by the informants (close relatives or caregivers of the subjects). Total ratings in both, 'MyCog' and 'TheirCog', parts range between 0 and 24, with higher ratings indicating greater perceived cognitive change.

\section{Apolipoprotein E Analysis}

Genomic DNA was extracted from peripheral blood of probands using the QIAamp DNA blood minikit (Qiagen AG, Basel, Switzerland). ApoE genotyping was performed by polymerase chain reaction amplification and HhaI restriction enzyme digestion. We classified the $\mathrm{CN}$ participants in two groups on the basis of the presence (carriers) or absence (noncarriers) of at least one ApoE4 allele. ApoE genotype results were not available for three participants (one for each group).

\section{Determination of CSF biomarkers}

All subjects underwent lumbar puncture in the morning (9-12 AM). CSF samples were centrifuged and stored in polypropylene tubes at $-80^{\circ} \mathrm{C}$. The $\mathrm{AB} 42$, tau, and p-tau at threonine 181 in CSF were measured by enzyme-linked immunosorbent assay (Innogenetics, Ghent, Belgium). Cut-off values of abnormality for each CSF biomarker were defined according to previous works (Antonell et al., 2011; van Harten et al., 2013): $\mathrm{AB} 42<550 \mathrm{pg} / \mathrm{ml}, \mathrm{tau}>450 \mathrm{pg} / \mathrm{ml}$, and $\mathrm{p}$-tau $>75 \mathrm{pg} / \mathrm{ml}$.

\section{Statistical methods}

Analysis of variance (ANOVA) was carried out to compare demographic/biological data means, and VMC task means in the CTR, the Pre-AD, and the AD groups using Bonferroni correction to adjust for multiple comparisons. Chi-square tests were employed to analyse categorical data. Independent sample t-tests were assessed to compare VMC task 
performance and standard neuropsychological performance in ApoE4 carriers and noncarriers. $T$-tests were also used to compare the performance shown by the three groups (AD patients, CTR, and Pre-AD) on each of the neuropsychological tests. Pearson's correlation was employed to evaluate the relationship between VMC task measures, including RTs, errors (responding with the wrong key), and omissions (no response), and the following variables: CSF biomarkers levels, neuropsychological performance, SCD-Q and HADS scores, age, and educational level (measured in years of education). Multiple regressions were used to explore the contribution of CSF biomarkers (AB42 and tau) and the ApoE 4 allele to VMC task outcome, that is how much of the variation in VMC measures can be explained by these three variables. We did not correct comparisons and correlations for age nor years of education, given that the VMC measures (RTs, errors and omissions) did not show correlations with any of them. Statistical significance was set at $p<.05$. All data were analysed using SPSS v20.0 (IBM Corp, Armonk, NY, USA).

\section{Results}

\section{Sample characteristics}

Means and standard deviations (SDs) for the demographic characteristics and all CSF biomarkers levels (AB42, tau, and p-tau) as well as ApoE4 allele distributions and psychological data (SCD-Q and HADS scores) are shown in Table 1. There were no statistically significant differences in terms of age and years of education between the CTR, Pre-AD, and AD groups. The AB42 levels were significantly different in the CTR group compared to both Pre-AD and AD participants $(p<.001)$. The CTR and the Pre-AD groups differed significantly in tau and p-tau levels from the AD group $(p<.001)$. ApoE4 distribution was significantly different in the CTR compared to both Pre-AD and AD groups $(p<.001)$. Furthermore, the CTR and Pre-AD groups did not show significant differences in neuropsychological measures (see details in Table 2). Similarly, the neuropsychological performance of the CN ApoE4 carriers did not differ significantly from the CN ApoE4 noncarriers (see details in Table 3). The performance of patients with $\mathrm{AD}$ on neuropsychological tests was also reported in Table 2.

\section{VMC task differences between CTR, Pre-AD, and AD}

A statistically significant difference was found in RTs $(\phi<.001)$ between the three tested groups (CTR, Pre-AD, and AD). Post hoc comparisons using Bonferroni test indicated that the RTs were, in average, significantly higher in Pre-AD compared to the CTR group $(p<.01)$. Furthermore, RTs in the CTR $(p<.001)$ and Pre-AD $(p<.05)$ groups were significantly faster than RTs in the AD group. Despite the fact that possible visuomotor difficulties can be observed in RTs but not in errors or omissions, further analyses were conducted considering these variables. The ANOVA showed a significant difference for errors (responding with the wrong key) but not for omissions (no response), between the three studied groups $(p<.01)$. Specifically, the number of errors was higher in the AD than in the CTR group $(p<.005)$. Means and SDs for the CTR, Pre-AD, and AD groups are detailed in Table 4.

\section{VMC task differences between ApoE4 carriers and noncarriers}

A significant difference was found between the group of CN carriers, which included four CTR and eight Pre-AD $(M=964.98, S D=169.44)$, and noncarriers, which included 42 


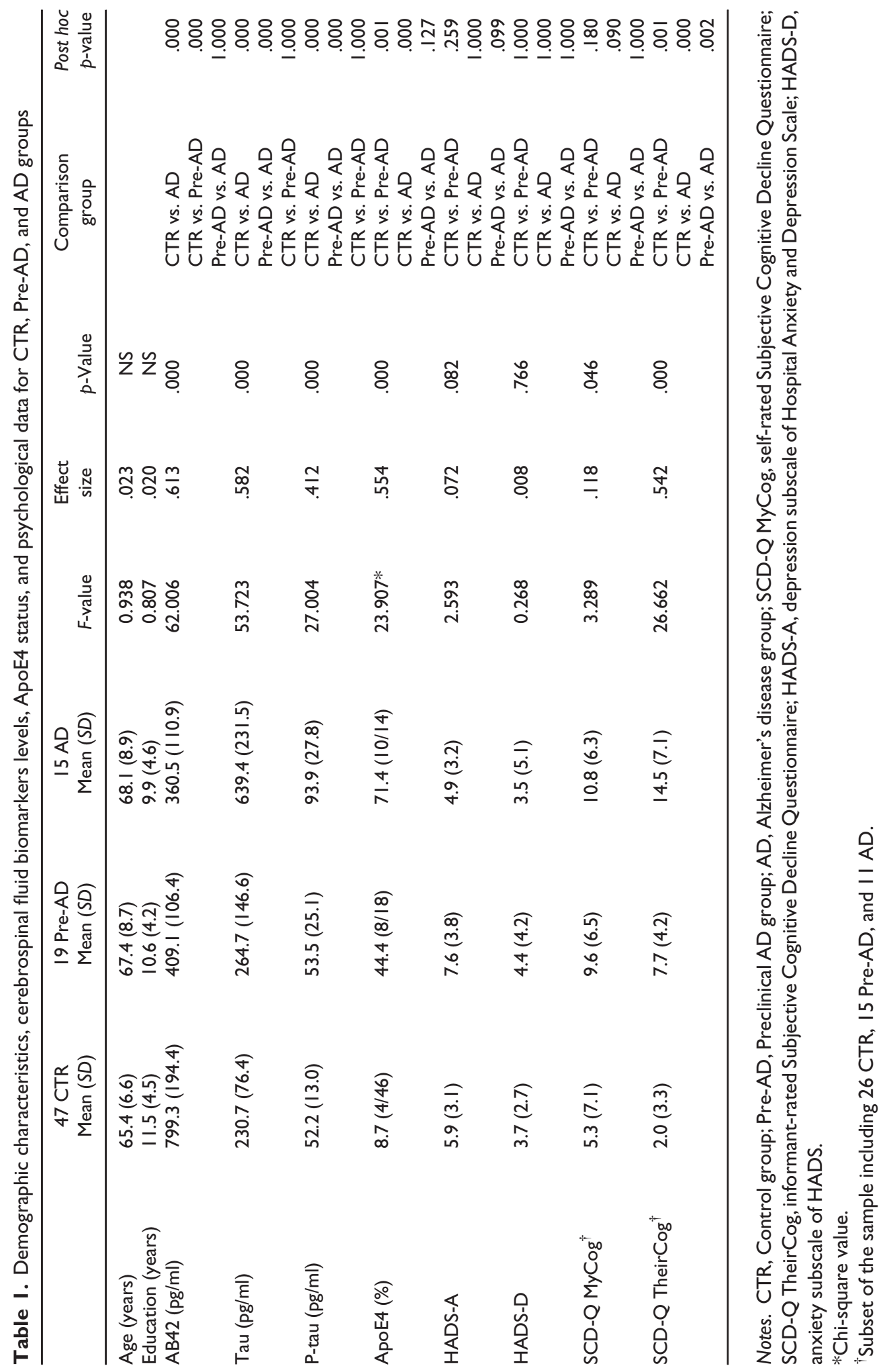


Table 2. Means (normalized scalar scores) of standard neuropsychological tests for CTR, Pre-AD, and AD groups

\begin{tabular}{|c|c|c|c|c|c|}
\hline & $\begin{array}{c}\text { CTR } \\
47 \\
\text { Mean (SD) }\end{array}$ & $\begin{array}{c}\text { Pre-AD } \\
19 \\
\text { Mean (SD) }\end{array}$ & $\begin{array}{c}A D \\
\text { I5 } \\
\text { Mean }(S D)\end{array}$ & Comparison groups & $p(<.05)$ \\
\hline FCSRT: Learning & II.6 (2.5) & II $.5(2.7)$ & $4.8(2.7)$ & $\begin{array}{l}\text { CTR vs. Pre-AD } \\
\text { Pre-AD vs. } A D \\
C T R \text { vs. } A D\end{array}$ & $\begin{array}{l}\text { NS } \\
.000 \\
.000\end{array}$ \\
\hline FCSRT: Total learning & $12.5(2.8)$ & $12.7(3.5)$ & $4.6(3.1)$ & $\begin{array}{l}\text { CTR vs. Pre-AD } \\
\text { Pre-AD vs. } A D \\
\text { CTR vs. AD }\end{array}$ & $\begin{array}{l}\text { NS } \\
.000 \\
.000\end{array}$ \\
\hline FCSRT: Recall & $11.9(2.5)$ & $11.6(2.3)$ & $2.9(1.8)$ & $\begin{array}{l}\text { CTR vs. Pre-AD } \\
\text { Pre-AD vs. } A D \\
\text { CTR vs. AD }\end{array}$ & $\begin{array}{l}\text { NS } \\
.000 \\
.000\end{array}$ \\
\hline FCSRT: Total recall & $14.0(3.9)$ & $12.7(4.6)$ & $4.7(3.5)$ & $\begin{array}{l}\text { CTR vs. Pre-AD } \\
\text { Pre-AD vs. } A D \\
\text { CTR vs. AD }\end{array}$ & $\begin{array}{l}\text { NS } \\
.000 \\
.000\end{array}$ \\
\hline Semantic fluency & $10.9(2.7)$ & $10.7(2.4)$ & $6.6(2.7)$ & $\begin{array}{l}C T R \text { vs. Pre-AD } \\
\text { Pre-AD vs. } A D \\
C T R \text { vs. } A D\end{array}$ & $\begin{array}{l}\text { NS } \\
.000 \\
.000\end{array}$ \\
\hline VOSP: Number location & I $3.6(4.2)$ & $12.9(4.8)$ & $7.6(4.4)$ & $\begin{array}{l}\text { CTR vs. Pre-AD } \\
\text { Pre-AD vs. } A D \\
C T R \text { vs. } A D\end{array}$ & $\begin{array}{l}\text { NS } \\
.007 \\
.000\end{array}$ \\
\hline TMT-part A & $10.5(2.9)$ & I0.I (1.6) & $8.0(1.6)$ & $\begin{array}{l}\text { CTR vs. Pre-AD } \\
\text { Pre-AD vs. } A D \\
\text { CTR vs. AD }\end{array}$ & $\begin{array}{l}\text { NS } \\
.001 \\
.008\end{array}$ \\
\hline Digit (WAIS-III): Direct & $9.9(1.5)$ & II.2(2.7) & $9.6(2.3)$ & $\begin{array}{l}\text { CTR vs. Pre-AD } \\
\text { Pre-AD vs. } A D \\
\text { CTR vs. AD }\end{array}$ & $\begin{array}{l}\text { NS } \\
\text { NS } \\
\text { NS }\end{array}$ \\
\hline Digit (WAIS-III): Inverse & $12.0(2.0)$ & II $.8(2.8)$ & $9.0(2.8)$ & $\begin{array}{l}\text { CTR vs. Pre-AD } \\
\text { Pre-AD vs. AD } \\
\text { CTR vs. AD }\end{array}$ & $\begin{array}{l}\text { NS } \\
.018 \\
.001\end{array}$ \\
\hline Vocabulary (WAIS-III)* & $13.3(3.5)$ & $12.3(2.4)$ & - & CTR vs. Pre-AD & NS \\
\hline Block design test (WAIS-III)* & $12.6(2.3)$ & I I.7 (2.7) & - & CTR vs. Pre-AD & NS \\
\hline VOSP: Object decision* & II.I (2.7) & $10.7(1.8)$ & - & CTR vs. Pre-AD & NS \\
\hline Boston Naming Test* & $11.4(2.2)$ & $11.2(2.6)$ & - & CTR vs. Pre-AD & NS \\
\hline TMT-part B* & $9.7(2.6)$ & $8.5(2.0)$ & - & CTR vs. Pre-AD & NS \\
\hline Stroop word* & $10.8(2.2)$ & $10.1(1.9)$ & - & CTR vs. Pre-AD & NS \\
\hline Stroop colour* & $10.5(2.5)$ & $9.9(3.0)$ & - & CTR vs. Pre-AD & NS \\
\hline Stroop colour-word* & II $.0(2.6)$ & $10.3(2.3)$ & - & CTR vs. Pre-AD & NS \\
\hline Digit Symbol (WAIS-III)* & $12.9(2.5)$ & $12.2(2.2)$ & - & CTR vs. Pre-AD & NS \\
\hline Symbol Digit Modalities Test* & $10.6(2.0)$ & I0.3 (2.7) & - & CTR vs. Pre-AD & NS \\
\hline
\end{tabular}

Notes. FCSRT, Free and Cued Selective Reminding Test; CTR, Control group; VOSP, Visual Object and Space Perception battery; WAIS, Wechsler Adult Intelligence Scale; TMT, Trail Making Test. *Neuropsychological tests administered to CTR and Pre-AD, but not to AD group.

CTR and 10 Pre-AD, $(M=855.37, S D=109.84)$ in RTs, $t(62)=-2.79, p=.007$, two-tailed, Cohen's $d=.76$ ) but not in errors or omissions. When comparing carriers and noncarriers within the Pre-AD group, no significant differences were found on any measure of the VMC task. 
Table 3. Means (normalized scalar scores) of standard neuropsychological tests for CN ApoE4 carriers and noncarriers

\begin{tabular}{lccc} 
& CN ApoE4 noncarriers & CN ApoE4 carriers & \\
& 52 & 12 & \\
& Mean $(S D)$ & Mean $(S D)$ & $p(<.05)$ \\
\hline Vocabulary (WAIS-III) & $13.0(2.9)$ & $12.8(3.9)$ & NS \\
Block design test (WAIS-III) & $12.5(2.3)$ & $12.0(3.1)$ & NS \\
FCSRT: Learning & $11.5(2.4)$ & $11.4(3.2)$ & NS \\
FCSRT: Total learning & $12.7(3.1)$ & $11.4(2.3)$ & NS \\
FCSRT: Recall & $11.9(2.5)$ & $11.5(2.3)$ & NS \\
FCSRT: Total recall & $13.7(3.9)$ & $12.9(5.3)$ & NS \\
Boston Naming Test & $11.6(2.3)$ & $10.6(2.4)$ & NS \\
Semantic fluency (animals) & $10.8(2.3)$ & $10.7(3.9)$ & NS \\
VOSP: Number location & $13.5(4.2)$ & $13.7(5.2)$ & NS \\
VOSP: Object decision & $10.6(2.1)$ & $12.0(3.6)$ & NS \\
TMT-part A & $10.5(2.6)$ & $10.2(2.5)$ & NS \\
TMT-part B & $9.6(2.3)$ & $8.9(3.3)$ & NS \\
Stroop word & $10.6(2.1)$ & $11.0(2.0)$ & NS \\
Stroop colour & $10.4(2.7)$ & $10.1(2.7)$ & NS \\
Stroop colour-word & $11.0(2.3)$ & $10.5(3.5)$ & NS \\
Digit Symbol (WAIS-III) & $13.1(2.3)$ & $11.7(2.5)$ & NS \\
Symbol Digit Modalities Test & $10.7(2.1)$ & $9.5(2.7)$ & NS \\
Digit (WAIS-III): Direct & $10.4(1.9)$ & $10.7(2.9)$ & NS \\
Digit (WAIS-III): Inverse & $12.2(2.3)$ & $11.0(2.2)$ & NS \\
\hline
\end{tabular}

Notes. CN ApoE4 noncarriers, cognitively normal individuals not carrying the ApoE4 allele; CN ApoE4 carriers, cognitively normal individuals carrying the ApoE4 allele; FCSRT, Free and Cued Selective Reminding Test; VOSP, Visual Object and Space Perception battery; WAIS, Wechsler Adult Intelligence Scale; TMT, Trail Making Test.

\section{Correlations between VMC task and CSF biomarkers (and demographic data)}

Considering the whole sample (i.e., including data from the CTR, the Pre-AD, and the AD groups), significant correlations were found between the RTs and CSF biomarkers $(p<.001$ for all biomarkers). Furthermore, when considering CN participants (CTR and Pre-AD), RTs correlated with CSF AB42 levels $(p<.01$; Figure 2), but not with tau or p-tau levels. Neither age nor years of education correlated with VMC measures in any of the tested groups, with the exception of a significant association found between RTs and years of education in AD group $(p<.05)$. Correlation coefficients of RTs with CSF biomarkers and demographic data are summarized in the Table 5. Analyses conducted for errors and omissions yielded a significant association exclusively between errors and p-tau levels $(r=.246, p<.031)$.

\section{Correlations between VMC task and cognitive/psychological measures}

Correlation coefficients between RTs and psychological measures are summarized in the Table 5. Significant positive correlations were found between RTs and informant-rated SCD measured by the SCD-Q TheirCog, when considering the whole sample $(p<.001)$ and also when restricting the analysis to $\mathrm{CN}(\phi<.01)$ and Pre-AD individuals $(p<.05)$. Moreover, RTs correlated with self-rated SCD measured by SCD-Q MyCog in the whole sample $(p<.05)$, but not with anxiety nor depression levels measured by HADS in any of 


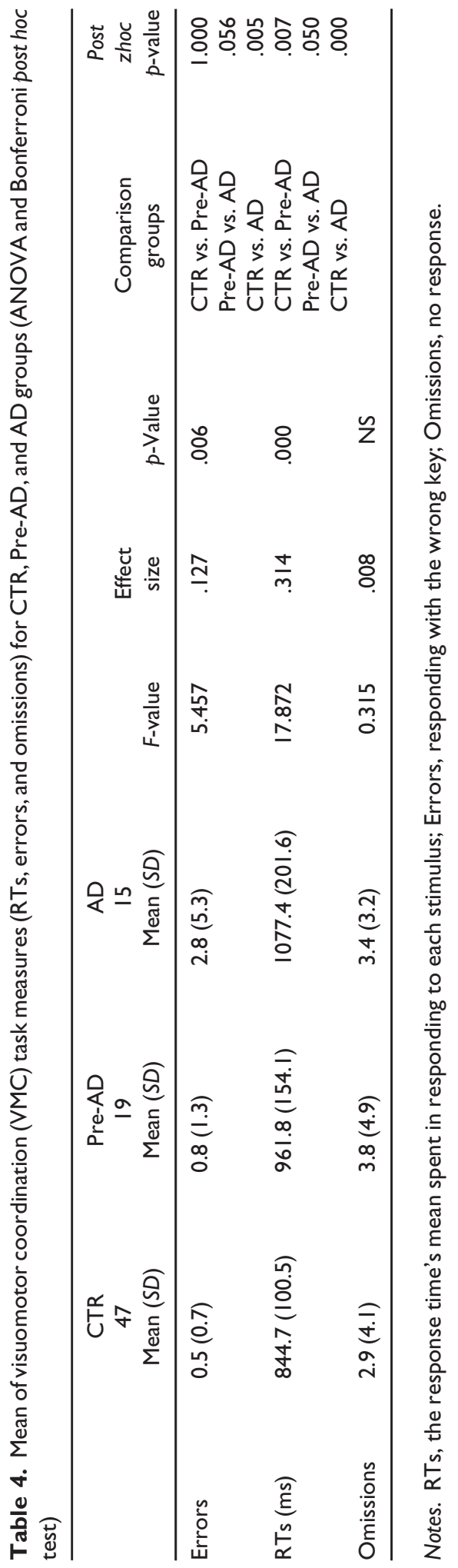




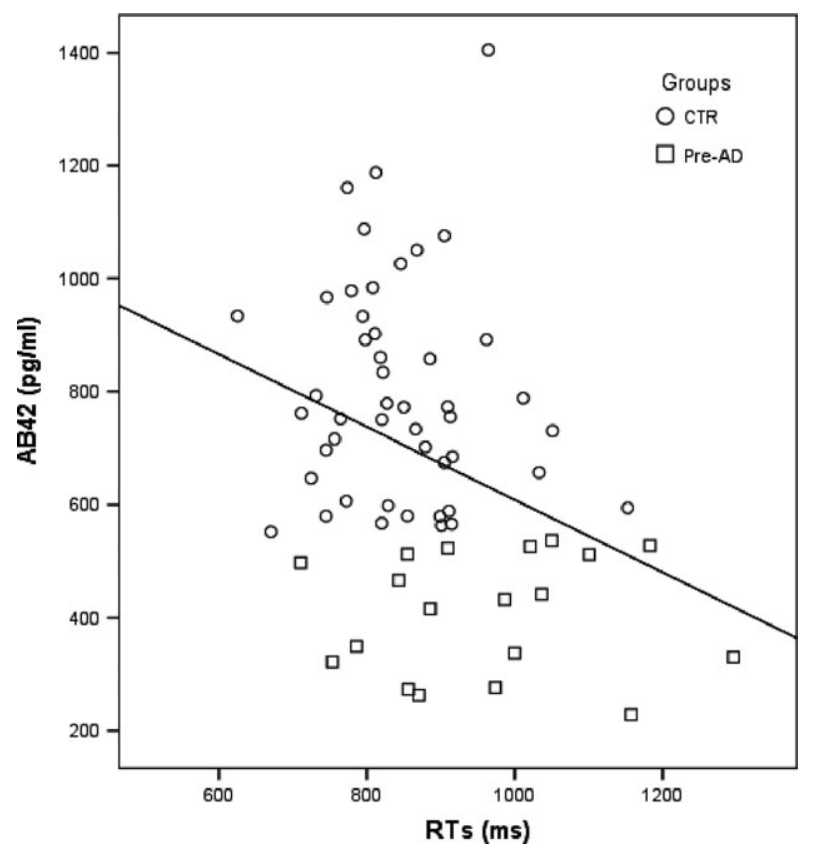

Figure 2. Scatter plot shows cerebrospinal fluid (CSF) AB42 levels and response times (RTs) of visuomotor coordination (VMC) task in CTR and Pre-AD groups.

Table 5. Pearson correlations of response times (RTs) with cerebrospinal fluid biomarkers levels, demographic characteristics, and psychological measures

\begin{tabular}{|c|c|c|c|c|c|c|c|c|c|c|}
\hline & \multicolumn{2}{|c|}{$\begin{array}{l}\text { Whole } \\
\text { sample } \\
(n=8 I)\end{array}$} & \multicolumn{2}{|c|}{$\mathrm{CN}(n=66)$} & \multicolumn{2}{|c|}{$\begin{array}{c}\text { CTR } \\
(n=47)\end{array}$} & \multicolumn{2}{|c|}{$\begin{array}{l}\text { Pre-AD } \\
(n=19)\end{array}$} & \multicolumn{2}{|c|}{$\mathrm{AD}(n=15)$} \\
\hline & $r$ & $p$ & $r$ & $p$ & $r$ & $p$ & $r$ & $p$ & $r$ & $p$ \\
\hline AB42 $(\mathrm{pg} / \mathrm{ml})$ & -.428 & .000 & -.333 & .006 & -.088 & .555 & .019 & .939 & .079 & .781 \\
\hline Tau (pg/ml) & .470 & .000 & .161 & .199 & .009 & .954 & .189 & .439 & .211 & .451 \\
\hline P-tau (pg/ml) & .393 & .000 & .071 & .576 & -.021 & .891 & .131 & .594 & .219 & .434 \\
\hline Age (years) & .105 & .349 & .222 & .073 & .153 & .303 & .235 & .334 & -.324 & .239 \\
\hline Education (years) & .011 & .922 & -.089 & .475 & .016 & .918 & -.188 & .441 & .548 & .034 \\
\hline HADS-A & -.051 & .675 & .131 & .326 & -.106 & .508 & .239 & .356 & -.292 & .358 \\
\hline HADS-D & -.043 & .722 & .116 & .386 & -.167 & .296 & .368 & .146 & -.303 & .338 \\
\hline SCD-Q MyCog* & .299 & .031 & .251 & .113 & .060 & .772 & .299 & .280 & .162 & .634 \\
\hline SCD-Q TheirCog* & .465 & .001 & .406 & .010 & -.152 & .478 & .600 & .018 & . 197 & .619 \\
\hline
\end{tabular}

Notes. Whole sample: CTR, Pre-AD and AD groups; CN, Cognitively normal participants; CTR, Control group, Pre-AD, Preclinical Alzheimer disease group; AD, Alzheimer's disease group; SCD-Q MyCog, selfrated Subjective Cognitive Decline Questionnaire; SCD-Q TheirCog, informant-rated Subjective Cognitive Decline Questionnaire; HADS-A, depression subscale of Hospital Anxiety and Depression Scale; HADS-D, anxiety subscale of Hospital Anxiety and Depression Scale.

*Subset of the sample including 26 CTR, I5 Pre-AD, and II AD individuals. 
the groups studied. Errors in VMC task also correlated with SCD-Q TheirCog ratings in the whole sample $(r=.395, p<.007)$. Regarding the cognitive performance on the neuropsychological battery, RTs correlated with the following tests in $\mathrm{CN}$ participants: The SDMT and the TMT-part B (both $p<.005$ ) and TMT-part A and Digit Symbol Coding (both $p<.05$; see Table S1). When analyses were conducted for errors and omissions, only deleted recall subtest of FCSRT showed a significant association with errors $(r=-.263, p<.035)$.

\section{Predictors of VMC task performance}

Standard multiple regression analysis was used to assess the ability of CSF biomarkers and the presence ApoE4 allele in predicting RTs in the VMC task. Because of collinearity among tau and p-tau biomarkers, we decided to include p-tau in the analysis, based on previous literature indicating that $\mathrm{p}$-tau is more sensitive and specific for $\mathrm{AD}$ (Koopman et al., 2009). When examining the whole sample, all variables, including p-tau levels $(\beta=.268, p=.010), \mathrm{AB} 42$ levels $(\beta=-.277, p=.011)$, and the ApoE4 allele $(\beta=.236$, $p=.032$ ), made a unique contribution to the model. When restricting the analysis to $\mathrm{CN}$ participants, the variables making a significant contribution to the prediction of RTs were AB42 levels $(\beta=-.279, p=.028)$ and the presence of ApoE4 allele $(\beta=.255, p=.042)$, but not p-tau levels. When conducting regression analysis separately for errors and omissions, the contributions of each of the independent variables studied were not significant.

\section{Discussion}

The new computerized VMC task employed in the present study discriminated Pre-AD from control participants, suggesting the presence of early visuomotor difficulties in Pre$\mathrm{AD}$ individuals. RTs of the VMC task were negatively correlated to CSF AB42 levels in CN individuals, and significantly higher in Pre-AD than in control group, even though these two groups did not present any difference in performance measured by standard tests. This pattern of results allows us to conclude that the new computerized VMC task is able to identify subtle cognitive difficulties in visuomotor coordination that cannot be detected otherwise. Moreover, CN subjects carrying the ApoE4 allele showed higher RTs than those without it in the VMC task. Besides, RTs positively correlated with informant-rated SCD, but not with self-rated SCD.

Cognitive performance has generally been considered inherently normal in the Pre-AD stage (Dubois et al., 2014). However, new efforts have been devoted to design new tests that can measure subtle cognitive difficulties at this stage (Rentz et al., 2013). In this context, computerized timed tests may offer additional benefits when measuring performance in Pre-AD individuals. Accordingly, performance in our VMC task was similar in the Pre-AD and CTR participants when measuring accuracy (as determined by the number of errors). Crucially, instead, these groups showed differences in their RTs. These results suggest that, while accuracy seems to be useful in detecting differences in patients with AD, RTs as obtained in speeded tasks may be more suitable for identifying less obvious differences between Pre-AD and CTR individuals.

Although $\mathrm{AD}$ is typically associated with impairments in memory and other aspects of cognition, it has been suggested that visuomotor function is equally impaired in patients with AD (de Boer, Mattace-Raso, van der Steen, \& Pel, 2014). Crucially, a subtle deterioration in visuomotor control has been observed, not only in patients with fully 
developed AD, but also at earlier stages (Tippett et al., 2012; Verheij et al., 2012). However, the exploration of visuomotor functioning in nondemented individuals is still scarce. Hawkins and Sergio (2014) proposed that visuomotor alterations are already present before the onset of dementia in individuals with increased risk of developing AD (participants with a family history or MCI were considered a unique AD risk group). However, the $\mathrm{AD}$ pathophysiological processes (including $\mathrm{AB}$ deposition) were not studied in this previous research. The present study went one step further and examined the possible relationship between visuomotor functioning and specific $\mathrm{AD}$ biomarkers in people with normal cognition as evaluated by several cognitive tests.

Higher RTs were associated with decreased CSF AB42 levels in CN individuals, but not with tau levels. Furthermore, when analysing the influence of ApoE 4 allele on the results observed in the VMC task, we found that CN ApoE4 carriers presented higher RTs than noncarriers, despite the fact that neuropsychological tests did not show any difference between them. In accordance, previous studies found that computerized measures of cognitive domains other than memory may be associated with the presence of ApoE4 allele (Espeseth et al., 2006; Reinvang, Winjevoll, Rootwelt, \& Espeseth, 2010). Moreover, multiple regression analyses confirmed that ApoE4 allele and AB42 levels were both predictors of RTs in CN participants.

Our findings suggest that visuomotor dysfunction occurs at a very early stage of the disease and may be associated with $\mathrm{AB} 42$ deposition, the neuropathological hallmark of $\mathrm{AD}$, and with ApoE4 allele, the major known genetic risk factor for sporadic $\mathrm{AD}$ (Lambert et al., 2013). However, further research is still needed to elucidate the relative impact of $\mathrm{AB} 42$ biomarker and ApoE4 allele on visuomotor performance in $\mathrm{CN}$ individuals.

As previously mentioned, standard cognitive measures of visuomotor components may present some limitations at detecting subtle difficulties in Pre-AD individuals. Based on our present results, performance on the VMC task was found to be associated with these standard measures, specifically to TMT, SDMT, and Digit Symbol Coding (see Results section). However, the performance on these tests was similar in the CTR and Pre-AD groups, and its relationship with $\mathrm{AD}$ biomarkers was not significant. In that respect, the relation between standard tests that assess visuomotor abilities and AD biomarkers still remains unclear (Hedden et al., 2013). One possible explanation for the lack of agreement in previous literature could be that standard tests of visuomotor abilities assess a broader range of cognitive domains, including executive functioning. In contrast, the VMC task introduced here was designed to tackle visuomotor coordination while reducing as much as possible engagement of other cognitive abilities. We suggest that applying adequate measures of specific functions may be crucial to find AB42-related dysfunctions in Pre-AD individuals.

Another possible advantage of the VMC task is its independence of compensatory mechanisms, such as educational level or cognitive reserve, considered a major confounder of the relationship between the cognitive performance and the $\mathrm{AD}$ pathophysiological processes (Rentz et al., 2010; Stern, 2009). Thus, while cognitive reserve is mostly associated with higher order executive functioning (Tucker \& Stern, 2011), it may have less impact on visuomotor coordination functions.

Our examination of possible links between SCD and the VMC task indicated that only informant-rated SCD, but not self-reported SCD, is associated with response slowness in the VMC task in $\mathrm{CN}$ participants. This finding is not surprising given that cognitive complaints are often related not only to memory problems, but also to other difficulties in everyday functioning (Amariglio et al., 2012; van Norden et al., 2008) which often involve visuomotor coordination abilities. Accordingly, a previous study described 
significant associations between informant-rated SCD and standard neuropsychological measures of visuomotor components in CN individuals (Rami et al., 2014). On the other hand, the nonsignificant correlation found between the VMC measure and the selfreported SCD-Q suggests that Pre-AD individuals might have some degree of unawareness of their subtle difficulties. This evidence is in line with previous literature on AD and anosognosia (Mograbi \& Morris, 2014). Our results are also consistent with previous literature showing that informant reports can be more reliable predictors than self-reports of subjects' cognitive performance, not only when referring to cognitively impaired individuals but also to individuals showing normal cognition as measured with standardized tests (Rabin et al., 2012; Rami et al., 2014; Slavin et al., 2010). Interestingly, the international Subjective Cognitive Decline Initiative (SCD-I) work group recently proposed the confirmation of SCD by others as an important feature for an increased risk of $\mathrm{AD}$ (Jessen et al., 2014).

Although the ability of the VMC task to correctly identify Pre-AD individuals is promising, neuroimaging studies would be needed to fully uncover the pathophysiological mechanisms underlying the visuomotor coordination anomalies observed in the Pre$\mathrm{AD}$ individuals. In this context, it should be noted that the VMC task has also a marked spatial attentional component, as participants have to attend to the location of the target on the computer screen and move their attentional focus towards the appropriate button of the keyboard. As confirmed in several imaging studies (Corbetta \& Shulman, 2002; Culham \& Valyear, 2006), the posterior parietal cortex is crucial in spatial attention and in integrating attention and motor systems. Interestingly, functional changes in the posterior parietal regions have been described in Pre-AD individuals (Hedden et al., 2009; Rami et al., 2012). Hence, it may be of particular importance to corroborate the possible association between the visuomotor anomalies, at a behavioural level, and the functional alterations found in parietal regions in early stages of AD.

A limitation of the current study could be the small sample size for the Pre-AD group, which could possibly limit our power to identify differences between ApoE 4 carriers and noncarriers within the Pre-AD group. Furthermore, the cross-sectional nature of this study precludes the possibility of testing the usefulness of the VMC task at predicting the subsequent cognitive decline in later stages of the disease. Moreover, although in the present study the VMC difficulties were found in otherwise asymptomatic individuals, we cannot rule out the possibility that subtle dysfunctions in domains other than visuomotor coordination could be observed, in these target individuals, with the use of more sensitive methods.

Overall, our results indicate that the VMC task is a sensitive tool for an early detection of subtle cognitive difficulties in Pre-AD stage and is related to $\mathrm{AB} 42$ levels in $\mathrm{CN}$ individuals. Moreover, the presence of ApoE4 allele and informant-rated SCD, considered both potential predictors of $\mathrm{AD}$, seems to be related to these visuomotor anomalies. Further research is required to evaluate visuomotor function as a sensitive marker for detecting the early effects of AB42 deposition and ApoE4 status. In conclusion, the knowledge derived from the present work can be used to develop new highly specific computerbased measures to detect subtle dysfunctions in Pre-AD individuals and model future preclinical trials.

\section{Acknowledgements}

Dr Lorena Rami is the recipient of a Miguel Servet Grant as a senior investigator (CP2/00023). This study was funded by the grants FIS PI11/01071 (Fondo Europeo de Desarrollo Regional, 
"una manera de hacer Europa") to Lorena Rami, and PSI2012-39149 from Ministerio de Economía y Competitividad (Spanish Government) to Jordi Navarra. We thank all volunteers for their participation in this study, without their collaboration this work would have not been possible. The authors report no biomedical financial interests or potential conflicts of interest.

\section{References}

Amariglio, R. E., Becker, J. A., Carmasin, J., Wadsworth, L. P., Lorius, N., Sullivan, C., . . Rentz, D. M. (2012). Subjective cognitive complaints and amyloid burden in cognitively normal older individuals. Neuropsychologia, 50, 2880-2886. doi:10.1016/j.neuropsychologia.2012.08.011

Antonell, A., Fortea, J., Rami, L., Bosch, B., Balasa, M., Sánchez-Valle, R., . . Lladó, A. (2011). Different profiles of Alzheimer's disease cerebrospinal fluid biomarkers in controls and subjects with subjective memory complaints. Journal of Neural Transmission, 118, 259-262. doi:10.1007/ s00702-010-0534-0

Buschke, H. (1984). Cued recall in Amnesia. Journal of Clinical and Experimental Neuropsychology, 6, 433-440. doi:10.1080/01688638408401233

Corbetta, M., \& Shulman, G. L. (2002). Control of goal-directed and stimulus-driven attention in the brain. Nature Reviews Neuroscience, 3, 201-215. doi:10.1038/nrn755

Culham, J. C., \& Valyear, K. (2006). Human parietal cortex in action. Current Opinion in Neurobiology, 16, 205-212. doi:10.1016/j.conb.2006.03.005

de Boer, C., Mattace-Raso, F., van der Steen, J., \& Pel, J. J. (2014). Mini-Mental State Examination subscores indicate visuomotor deficits in Alzheimer's disease patients: A cross-sectional study in a Dutch population. Geriatrics \& Gerontology International, 14, 880-885. doi:10.1111/ ggi. 12183

Donohue, M. C., Sperling, R. A., Salmon, D. P., Rentz, D. M., Raman, R., Thomas, R. G., ... Alzheimer's Disease Cooperative Study (2014). Australian Imaging, Biomarkers, and Lifestyle Flagship Study of Ageing, the Alzheimer's Disease Neuroimaging Initiative, and the Alzheimer's Disease Cooperative Study. The preclinical Alzheimer cognitive composite: Measuring amyloidrelated decline. JAMA Neurology, 71, 961-970. doi:10.1001/jamaneurol.2014.803

Dubois, B., Feldman, H. H., Jacova, C., Hampel, H., Molinuevo, J. L., Blennow, K., . . Cummings, J. L. (2014). Advancing research diagnostic criteria for Alzheimer's disease: The IWG-2 criteria. Lancet Neurology, 13, 614-629. doi:10.1016/S1474-4422(14)70090-0

Espeseth, T., Greenwood, P. M., Reinvang, I., Fjell, A. M., Walhovd, K. B., Westlye, L. T., ... Parasuraman, R. (2006). Interactive effects of APOE and CHRNA4 on attention and white matter volume in healthy middle-aged and older adults. Cognitive, Affective, \& Behavioral Neuroscience, 6, 31-43. doi:10.3758/CABN.6.1.31

Ewers, M., Brendel, M., Rizk-Jackson, A., Rominger, A., Bartenstein, P., Schuff, N., ... Alzheimer's Disease Neuroimaging Initiative (ADNI) (2014). Reduced FDG-PET brain metabolism and executive function predict clinical progression in elderly healthy subjects. NeuroImage: Clinical, 4, 45-52. doi: 10.1016/j.nicl.2013.10.018

Forster, K. L., \& Forster, J. C. (2003). DMDX: A Windows display program with millisecond accuracy. Behavior Research Methods, Instruments, \& Computers, 35, 116-124. doi:10.3758/ bf03195503

Golden, C. (1978). Stroop color and word test. Chicago, IL: Stoelting.

Hawkins, K. M., \& Sergio, L. E. (2014). Visuomotor impairments in older adults at increased Alzheimer's disease risk. Journal of Alzheimer's Disease, 42, 607-621. doi:10.3233/JAD140051

Hedden, T., Oh, H., Younger, A. P., \& Patel, T. A. (2013). Meta-analysis of amyloid-cognition relations in cognitively normal older adults. Neurology, 80, 1341-1348. doi:10.1212/WNL. 0b013e31828ab35d 
Hedden, T., Van Dijk, K. R., Becker, J. A., Mehta, A., Sperling, R. A., Johnson, K. A., \& Buckner, R. L. (2009). Disruption of functional connectivity in clinically normal older adults harboring amyloid burden. Journal of Neuroscience, 29, 12686-12694. doi:10.1523/JNEUROSCI. 3189-09.2009

Jack, Jr, C. R., Knopman, D. S., Jagust, W. J., Shaw, L. M., Aisen, P. S., Weiner, M. W., \& Trojanowski, J. Q. (2010). Hypothetical model of dynamic biomarkers of the Alzheimer's pathological cascade. Lancet Neurology, 9, 119-128. doi:10.1016/S1474-4422(09)70299-6

Jack, Jr, C. R., Knopman, D. S., Weigand, S. D., Wiste, H. J., Vemuri, P., Lowe, V., . . Petersen, R. C. (2012). An operational approach to National Institute on Aging-Alzheimer's Association criteria for preclinical Alzheimer disease. Annals of Neurology, 71, 765-775. doi:10.1002/ana.22628

Jessen, F., Amariglio, R. E., van Boxtel, M., Breteler, M., Ceccaldi, M., Chátelat, G., ... Subjective Cognitive Decline Initiative (SCD-I) Working Group (2014). A conceptual framework for research on subjective cognitive decline in preclinical Alzheimer's disease. Alzheimer's $\&$ Dementia, 10, 844-852. doi:10.1016/j.jalz.2014.01.001

Kaplan, E., Goodglass, H., \& Weintraub, S. (1983). The Boston naming test. Philadelphia, PA: Lea \& Febiger.

Koopman, K., Le Bastard, N., Martin, J. J., Nagels, G., De Deyn, P. P., \& Engelborghs, S. (2009). Improved discrimination of autopsy-confirmed Alzheimer's disease (AD) from non-AD dementias using CSF P-tau181P. Neurochemistry International, 55, 214-218. doi:10.1016/ j.neuint.2009.02.017

La Joie, R., Perrotin, A., Barré, L., Hommet, C., Mézenge, F., Ibazizene, M., ... Chételat, G. (2012). Region-specific hierarchy between atrophy, hypometabolism, and $\beta$-amyloid (A $\beta$ ) load in Alzheimer's disease dementia. Journal of Neuroscience, 32, 16265-16273. doi:10.1523/ JNEUROSCI.2170-12.2012

Lambert, J. C., Ibrahim-Verbaas, C. A., Harold, D., Naj, A. C., Sims, R., Bellenguez, C., . . Amouyel, P. (2013). Meta-analysis of 74,046 individuals identifies 11 new susceptibility loci for Alzheimer's disease. Nature Genetics, 45, 1452-1458. doi:10.1038/ng.2802

Mandal, P. K., Joshi, J., \& Saharan, S. (2012). Visuospatial perception: An emerging biomarker for Alzheimer's disease. Review. Journal of Alzheimer's Disease, 3, S117-S135. doi:10.3233/JAD2012-120901

Mograbi, D. C., \& Morris, R. G. (2014). The developing concept of implicit awareness: A rejoinder and reply to commentaries on Mograbi and Morris (2013). Cognitive Neuroscience, 5, 138-142. doi:10.1080/17588928.2014.905522

Mormino, E. C., Betensky, R. A., Hedden, T., Schultz, A. P., Ward, A., Huijbers, W., . . Harvard Aging Brain Study (2014). Amyloid and APOE 4 interact to influence short-term decline in preclinical Alzheimer disease. Neurology, 82, 1760-1767. doi:10.1212/WNL.0000000000000431

Parra, M. A., Sala, S. D., Abrahams, S., Logie, R. H., Mendez, L. G., \& Lopera, F. (2011). Specific deficit of colour-colour short-term memory binding in sporadic and familial Alzheimer's disease. Neuropsychologia, 49, 1943-1952. doi:10.1016/j.neuropsychologia.2011.03.022

Peña-Casanova, J., Aguilar, M., Santacruz, P., Bertran-Serra, I., Hernández, G., Sol, J. M., . . Blesa, R. (1997). Adaptation and normalization of the Alzheimer's disease Assessment Scale for Spain (NORMACODEM) (II). Neurologia, 12, 69-77.

Perrotin, A., Mormino, E. C., Madison, C. M., Hayenga, A. O., \& Jagust, W. J. (2012). Subjective cognition and amyloid deposition imaging: A Pittsburgh Compound B positron emission tomography study in normal elderly individuals. Archives of Neurology, 69, 223-229. doi:10.1001/archneurol.2011.666

Rabin, L. A., Wang, C., Katz, M. J., Derby, C. A., Buschke, H., \& Lipton, R. B. (2012). Predicting Alzheimer's disease: Neuropsychological tests, self-reports, and informant reports of cognitive difficulties. Journal of the American Geriatrics Society, 60, 1128-1134. doi:10.1111/j.15325415.2012.03956.x

Rami, L., Mollica, M. A., García-Sanchez, C., Saldaña, J., Sanchez, B., Sala, I., .. Molinuevo, J. L. (2014). The Subjective Cognitive Decline Questionnaire (SCD-Q): A validation study. Journal of Alzheimer's Disease, 41, 453-466. doi:10.3233/JAD-132027 
Rami, L., Sala-Llonch, R., Solé-Padullés, C., Fortea, J., Olives, J., Lladó, A., . . Molinuevo, J. L. (2012). Distinct functional activity of the precuneus and posterior cingulate cortex during encoding in the preclinical stage of Alzheimer's disease. Journal of Alzheimer's Disease, 31, 517-526. doi:10.3233/JAD-2012-120223

Reinvang, I., Winjevoll, I. L., Rootwelt, H., \& Espeseth, T. (2010). Working memory deficits in healthy APOE epsilon 4 carriers. Neuropsychologia, 48, 566-573. doi:10.1016/j.neuropsy chologia.2009.10.018

Reitan, R. M., \& Wolfson, D. (1985). The Halstead-Reitan neuropsycholgical test battery: Therapy and clinical interpretation. Tucson, AZ: Neuropsychological Press. doi:10.1016/j.neuropsy chologia.2009.10.018

Rentz, D. M., Amariglio, R. E., Becker, J. A., Frey, M., Olson, L. E., Frishe, K., . . Sperling, R. A. (2011). Face-name associative memory performance is related to amyloid burden in normal elderly. Neuropsychologia, 49, 2776-2783. doi:10.1016/j.neuropsychologia.2011.06.006

Rentz, D. M., Locascio, J. J., Becker, J. A., Moran, E. K., Eng, E., Buckner, R. L., . . Johnson, K. A. (2010). Cognition, reserve, and amyloid deposition in normal aging. Annals of Neurology, 67, 353-364. doi:10.1002/ana.21904

Rentz, D. M., Parra Rodriguez, M. A., Amariglio, R., Stern, Y., Sperling, R., \& Ferris, S. (2013). Promising developments in neuropsychological approaches for the detection of preclinical Alzheimer's disease: A selective review. Alzheimer's Research \& Therapy, 5, 58. doi:10.1186/ alzrt222

Slavin, M. J., Brodaty, H., Kochan, N. A., Crawford, J. D., Trollor, J. N., Draper, B., \& Sachdev, P. S. (2010). Prevalence and predictors of "subjective cognitive complaints" in the Sydney Memory and Ageing Study. American Journal of Geriatric Psychiatry, 18, 701-710. doi:10.1097/ JGP.0b013e3181df49fb

Smith, A. (1982). Symbol digits modalities test. Los Angeles, CA: Western Psychological Services.

Snitz, B. E., Weissfeld, L. A., Lopez, O. L., Kuller, L. H., Saxton, J., Singhabahu, D. M., . . Dekosky, S. T. (2013). Cognitive trajectories associated with b-amyloid deposition in the oldest-old without dementia. Neurology, 80, 1378-1384. doi:10.1212/WNL.0b013e31828c2fc8

Sperling, R. A., Aisen, P. S., Beckett, L. A., Bennett, D. A., Craft, S., Fagan, A. M., ... Phelps, C. H. (2011). Toward defining the preclinical stages of Alzheimer's disease: Recommendations from the National Institute on Aging-Alzheimer's Association workgroups on diagnostic guidelines for Alzheimer's disease. Alzheimer's \& Dementia, 7, 280-292. doi:10.1016/j.jalz.2011.03.003

Stern, Y. (2009). Cognitive reserve. Neuropsychologia, 47, 2015-2028. doi:10.1016/ j.neuropsychologia.2009.03.004

Tippett, W. J., Sergio, L. E., \& Black, S. E. (2012). Compromised visually guided motor control in individuals with Alzheimer's disease: Can reliable distinctions be observed? Journal of Clinical Neuroscience, 19, 655-660. doi:10.1016/j.jocn.2011.09.013

Tucker, A. M., \& Stern, Y. (2011). Cognitive reserve in aging. Current Alzheimer Research, 8, 354 360. doi:10.2174/156720511795745320

van Harten, A. C., Smits, L. L., Teunissen, C. E., Visser, P. J., Koene, T., Blankenstein, M. A., . . van der Flier, W. M. (2013). Preclinical AD predicts decline in memory and executive functions in subjective complaints. Neurology, 81, 1409-1416. doi:10.1212/WNL.0b013e3182a8418b

van Norden, A. G., Fick, W. F., de Laat, K. F., van Uden, I. W., van Oudheusden, L. J., Tendolkar, I., . . . de Leeuw, F. E. (2008). Subjective cognitive failures and hippocampal volume in elderly with white matter lesions. Neurology, 71, 1152-1159. doi:10.1212/01.wnl.0000327564. 44819.49

Verheij, S., Muilwijk, D., Pel, J. J., van der Cammen, T. J., Mattace-Raso, F. U., \& van der Steen, J. (2012). Visuomotor impairment in early-stage Alzheimer's disease: Changes in relative timing of eye and hand movements. Journal of Alzheimer's Disease, 30, 131-143. doi:10.3233/JAD-2012111883

Warrington, E. K., \& James, M. (1986). Visual object recognition in patients with right-hemisphere lesions: Axes or features? Perception, 15, 355-366. doi:10.1068/p150355 
Wechsler, D. (1997). Wechsler adult intelligence scale (3rd ed.). San Antonio, TX: Psychological Corporation.

Zigmond, A. S., \& Snaith, R. P. (1983). The hospital anxiety and depression scale. Acta Psychiatrica Scandinavica, 67, 361-370. doi:10.1111/j.1600-0447.1983.tb09716.x

Received 17 February 2015

\section{Supporting Information}

The following supporting information may be found in the online edition of the article:

Table S1. Correlations between response time (RTs) of visuomotor coordination (VMC) task and standard neuropsychological tests. 